\title{
Existence of Different Intermediate Hamiltonians in Type A $\mathcal{N}$-fold Supersymmetry
}

\author{
Bijan Bagchi* \\ Department of Applied Mathematics, University of Calcutta, \\ 92 Acharya Prafulla Chandra Road, Kolkata 700 009, India. \\ Toshiaki Tanaka ${ }^{\dagger}$ \\ Department of Physics, \\ National Cheng Kung University, \\ Tainan 701, Taiwan, R.O.C. \\ National Center for Theoretical Sciences, \\ Taiwan, R.O.C.
}

\begin{abstract}
Type A $\mathcal{N}$-fold supercharge admits a one-parameter family of factorizations into product of $\mathcal{N}$ first-order linear differential operators due to an underlying $G L(2, \mathbb{C})$ symmetry. As a consequence, a type $\mathrm{A} \mathcal{N}$-fold supersymmetric system can have different intermediate Hamiltonians corresponding to different factorizations. We derive the necessary and sufficient conditions for the latter system to possess intermediate Hamiltonians for the $\mathcal{N}=2$ case. We then show that whenever it has (at least) one intermediate Hamiltonian, it can admit second-order parasupersymmetry and a generalized 2-fold superalgebra. As an illustration, we construct a set of generalized Pöschl-Teller potentials of this kind.
\end{abstract}

PACS numbers: 03.65.Ca; 03.65.Fd; 11.30.Na; 11.30.Pb

Keywords: $\mathcal{N}$-fold supersymmetry; Parasupersymmetry; Factorization method; Intertwining operators; Pöschl-Teller potentials

*Electronic address: bbagchi123@rediffmail.com

${ }^{\dagger}$ Electronic address: ttanaka@mail.ncku.edu.tw 


\section{INTRODUCTION}

Supersymmetric quantum mechanics (SUSY QM) has constituted an active research field in theoretical sciences over two decades. Although the original motivation for studying SUSY QM was to unveil the mechanisms of its dynamical breaking in quantum field theories [1], it turned out that SUSY QM, as the minimum building block of SUSY, contains various relevant concepts which provide convenient platforms to uncover many useful properties of quantum mechanics [2, 3, 4]. In particular, it is consistent with factorization schemes [5] and intertwining relationships [6] thereby providing a powerful tool to construct solvable Schrödinger equations. Furthermore, interesting extensions to higher-order SUSY schemes were carried out by taking recourse to higher-derivative versions of the factorization operators $[7,8,9]$.

The original extension in Ref. [7] was considered by using higher-order intertwining operators which are expressed as products of first-order linear differential operators and then applying the ordinary SUSY results. Later in Refs. [10,11], through the analysis of the general second-order case, the concept of reducibility was introduced. In this regard, a higher-order intertwining operator is said to be reducible if it is factorized into a product of first-order differential operators such that with respect to each factor there exists an intermediate real Hamiltonian satisfying a (shifted) SUSY relation. Otherwise it is called irreducible. This concept, however, seems less useful in view of the current status where non-Hermitian quantum theories have been investigated intensively since the discovery of $\mathcal{P} \mathcal{T}$ symmetry [12]. In fact, the SUSY method turned to be useful also in constructing a complex potential with real spectrum $[13,14,15]$.

In addition to the usefulness of the reality constraint, there arises a natural question about the well-definiteness of the concept if we take into account the fact that in general factorization of higher-order linear differential operators is not unique. The latter fact indeed has been reported in the context of the factorization method and ordinary SUSY QM, that is, there exist several Schrödinger operators that admit different factorizations [16, 17, 18, 19, 20, 21, 22], see also Ref. [23] for a recent approach. Hence, a reducible higher-order intertwining operator may admit simultaneously another factorization for which there are no intermediate real Hamiltonians.

On the other hand, the non-uniqueness in factorizing intertwining operators of arbitrary finite orders was, to the best of our knowledge, first reported in Ref. [24] for the well-known quasi-solvable sextic anharmonic oscillator potentials in the framework of type A $\mathcal{N}$-fold supersymmetry (see, Eq. (47) in the latter reference). Later it was shown that the nonuniqueness of factorizations in type A $\mathcal{N}$-fold SUSY is a consequence of the underlying $G L(2, \mathbb{C})$ symmetry [25].

In a recent communication [26], the following two-parameter family of second-order supersymmetric (SSUSY) system $\left(h^{(1)}, h^{(2)}, \hat{A} \hat{B}\right)$, characterized by the two parameters $A$ and $B$, satisfying $\hat{A} \hat{B} h^{(1)}=h^{(2)} \hat{A} \hat{B}$ was constructed:

$$
\begin{aligned}
& h^{(1)}=\hat{B}^{\dagger} \hat{B}+\frac{\bar{c}}{2}=-\frac{\mathrm{d}^{2}}{\mathrm{~d} x^{2}}+V_{A, B}(x)-\tilde{E}+\frac{\bar{c}}{2}, \\
& h^{(2)}=\hat{A} \hat{A}^{\dagger}-\frac{\bar{c}}{2}=-\frac{\mathrm{d}^{2}}{\mathrm{~d} x^{2}}+V_{A, B, \operatorname{ext}}(x)-E-\frac{\bar{c}}{2},
\end{aligned}
$$


where

$$
\begin{aligned}
V_{A, B}(x) & =\left[B^{2}+A(A+1)\right] \operatorname{csch}^{2} x-B(2 A+1) \operatorname{csch} x \operatorname{coth} x \\
V_{A, B, \text { ext }}(x) & =V_{A, B}(x)+\frac{2(2 A+1)}{2 B \cosh x-2 A-1}-\frac{2\left[4 B^{2}-(2 A+1)^{2}\right]}{(2 B \cosh x-2 A-1)^{2}},
\end{aligned}
$$

and the constants $E, \tilde{E}$, and $\bar{c}$ are given by

$$
E=-\left(B \mp \frac{1}{2}\right)^{2}, \quad \tilde{E}=-\left(B \pm \frac{1}{2}\right)^{2}, \quad \bar{c}=\mp 2 B .
$$

The intertwining operators $\hat{A}$ and $\hat{B}$ are respectively given by

$$
\begin{aligned}
& \hat{A}=\frac{\mathrm{d}}{\mathrm{d} x} \pm\left(B \pm \frac{1}{2}\right) \operatorname{coth} x \mp\left(A+\frac{1}{2}\right) \operatorname{csch} x-\frac{2 B \sinh x}{2 B \cosh x-2 A-1} \\
& \hat{B}=\frac{\mathrm{d}}{\mathrm{d} x} \mp\left(B \pm \frac{1}{2}\right) \operatorname{coth} x \pm\left(A+\frac{1}{2}\right) \operatorname{csch} x
\end{aligned}
$$

It was further shown in Ref. [26] that the system admits the intermediate Hamiltonians $h$ given by

$$
\begin{aligned}
h & =\hat{A}^{\dagger} \hat{A}-\frac{\bar{c}}{2}=-\frac{\mathrm{d}^{2}}{\mathrm{~d} x^{2}}+V_{A, B \pm 1}(x)-E-\frac{\bar{c}}{2} \\
& =\hat{B} \hat{B}^{\dagger}+\frac{\bar{c}}{2}=-\frac{\mathrm{d}^{2}}{\mathrm{~d} x^{2}}+V_{A, B \pm 1}(x)-\tilde{E}+\frac{\bar{c}}{2}
\end{aligned}
$$

with

$$
V_{A, B \pm 1}(x)=\left[(B \pm 1)^{2}+A(A+1)\right] \operatorname{csch}^{2} x-(B \pm 1)(2 A+1) \operatorname{csch} x \operatorname{coth} x
$$

which satisfy the ordinary SUSY relations $\hat{A} h=h^{(2)} \hat{A}$ and $\hat{B} h^{(1)}=h \hat{B}$. We shall hereafter call the system (1)-(9) the BQR SSUSY model.

Taking into account the fact that type A 2-fold SUSY is the necessary and sufficient condition for the existence of (at least) two linearly independent analytic (local) solutions to Schrödinger equation of one degree of freedom [27], we immediately know that the above BQR SSUSY model, which is exactly solvable, also belongs to type A 2-fold SUSY. Therefore, it would be natural that it has (at least) two different factorizations of the second-order intertwining operator in view of the aforementioned $G L(2, \mathbb{C})$ symmetry and has two different intermediate Hamiltonians correspondingly.

Regarding the existence of intermediate Hamiltonians, we note the following fact shown in Ref. [24] that the most general form of type A $\mathcal{N}$-fold SUSY quantum systems constructed directly from the $\mathcal{N}$ th-order intertwining operators of type A, namely, type A $\mathcal{N}$-fold supercharge $P_{\mathcal{N}}^{-}=P_{\mathcal{N} 1}^{-} \ldots P_{\mathcal{N} \mathcal{N}}^{-}$(cf., Eq. (11)) by solving $P_{\mathcal{N}}^{-} H^{-}=H^{+} P_{\mathcal{N}}^{-}$is more general than that of the systems constructed from the $\mathcal{N}$ repeated applications of the first-order intertwining operators $P_{\mathcal{N} k}^{-}$by solving $P_{\mathcal{N} k}^{-} H^{(k-1)}=H^{(k)} P_{\mathcal{N} k}^{-}(k=1, \ldots, \mathcal{N})$ with the identification $H^{-}=H^{(0)}$ and $H^{+}=H^{(\mathcal{N})}$. It is apparent that in the latter construction we automatically obtain a series of the intermediate Hamiltonians $H^{(1)}, \ldots, H^{(\mathcal{N}-1)}$ in addition to the $\mathcal{N}$-fold SUSY pair Hamiltonians $H^{ \pm}$at the cost of the generality. In the former construction, on 
the other hand, the existence of intermediate Hamiltonians is not guaranteed in general. Hence, the fact that the type A $\mathcal{N}$-fold supercharge has the factorized form by definition does not necessarily imply the existence of intermediate Hamiltonians. In particular, the fact that type A $\mathcal{N}$-fold supercharge admits different factorizations does not automatically mean that the most general type A $\mathcal{N}$-fold SUSY system has different sets of intermediate Hamiltonians accordingly.

Motivated by the backgrounds described above, we investigate in this article under what conditions type A $\mathcal{N}$-fold SUSY systems admit intermediate Hamiltonians in the case of $\mathcal{N}=2$. Furthermore, we also examine under the satisfaction of the conditions how many sets of such Hamiltonians are admissible for the type A 2-fold SUSY systems. In addition, we show that any such a system has another type of nonlinear supersymmetries, namely, parasupersymmetry of order 2 [28].

We organize the article as follows. In the next section, we review the framework of type A $\mathcal{N}$-fold SUSY by putting emphasis on the $G L(2, \mathbb{C})$ symmetry. In Section III, we investigate in details under what conditions a type A 2-fold SUSY quantum system has one or more intermediate Hamiltonians. In particular, we show that the maximum number of different intermediate Hamiltonians in type A 2-fold SUSY is two. In Section IV, we further show that when a type A 2-fold SUSY system admits (at least) one intermediate Hamiltonian, the system can have second-order parasupersymmetry. A novel generalization of 2-fold superalgebra is discussed briefly. As an application of the results, we construct in Section V a type A 2-fold SUSY system with two different intermediate Hamiltonians of generalized Pöschl-Teller type which includes the BQR SSUSY model as a particular case. Then, we close the article with discussion and perspectives of further developments in the last section.

\section{TYPE A $\mathcal{N}$-FOLD SUPERSYMMETRY AND $G L(2, \mathbb{C})$ COVARIANCE}

Roughly speaking, type A $\mathcal{N}$-fold SUSY quantum systems are composed of a pair of scalar Hamiltonians $H^{ \pm}$and an $\mathcal{N}$ th-order linear differential operator $P_{\mathcal{N}}^{-}$of the following forms: ${ }^{1}$

$$
\begin{gathered}
H^{ \pm}=-\frac{1}{2} \frac{\mathrm{d}^{2}}{\mathrm{~d} x^{2}}+\frac{1}{2} W(x)^{2}-\frac{\mathcal{N}^{2}-1}{24}\left(2 E^{\prime}(x)-E(x)^{2}\right) \pm \frac{\mathcal{N}}{2} W^{\prime}(x)-R, \\
P_{\mathcal{N}}^{-}=\prod_{k=0}^{\mathcal{N}-1}\left(\frac{\mathrm{d}}{\mathrm{d} x}+W(x)+\frac{\mathcal{N}-1-2 k}{2} E(x)\right),
\end{gathered}
$$

where $R$ is a constant while $E(x)$ and $W(x)$ are analytic functions satisfying

$$
\begin{array}{r}
\left(\frac{\mathrm{d}}{\mathrm{d} x}-E(x)\right) \frac{\mathrm{d}}{\mathrm{d} x}\left(\frac{\mathrm{d}}{\mathrm{d} x}+E(x)\right) W(x)=0 \text { for } \quad \mathcal{N} \geq 2, \\
\left(\frac{\mathrm{d}}{\mathrm{d} x}-2 E(x)\right)\left(\frac{\mathrm{d}}{\mathrm{d} x}-E(x)\right) \frac{\mathrm{d}}{\mathrm{d} x}\left(\frac{\mathrm{d}}{\mathrm{d} x}+E(x)\right) E(x)=0 \text { for } \quad \mathcal{N} \geq 3 .
\end{array}
$$

\footnotetext{
${ }^{1}$ We keep the original notations as far as possible. Thus, note that the function $E(x)$ is different from the constant $E$ in the BQR SSUSY model (1)-(9). Similarly, the function $A(z)$ introduced later in (19) is different from the parameter $A$ in the latter model.
} 
The product of operators appeared in (11) is defined by

$$
\prod_{k=0}^{n} A_{k} \equiv A_{n} \ldots A_{1} A_{0} .
$$

The operators $H^{ \pm}$and $P_{\mathcal{N}}^{-}$satisfy an intertwining relation

$$
P_{\mathcal{N}}^{-} H^{-}=H^{+} P_{\mathcal{N}}^{-}
$$

One of the most important features of type A $\mathcal{N}$-fold SUSY quantum systems is that the gauged Hamiltonians $\tilde{H}^{-}$and $\bar{H}^{+}$introduced by

$$
\overline{\tilde{H}}^{ \pm}=\mathrm{e}^{\mathcal{W}_{\mathcal{N}}^{ \pm}} H^{ \pm} \mathrm{e}^{-\mathcal{W}_{\mathcal{N}}^{ \pm}}, \quad \mathcal{W}_{\mathcal{N}}^{ \pm}(x)=\frac{\mathcal{N}-1}{2} \int \mathrm{d} x E(x) \mp \int \mathrm{d} x W(x),
$$

preserve the so-called type A monomial space $\tilde{\mathcal{V}}_{\mathcal{N}}^{(\mathrm{A})}$ :

$$
\overline{\tilde{H}}^{ \pm} \tilde{\mathcal{V}}_{\mathcal{N}}^{(\mathrm{A})} \subset \tilde{\mathcal{V}}_{\mathcal{N}}^{(\mathrm{A})}, \quad \tilde{\mathcal{V}}_{\mathcal{N}}^{(\mathrm{A})}=\left\langle 1, z(x), \ldots, z(x)^{\mathcal{N}-1}\right\rangle
$$

where the new variable $z(x)$ satisfies

$$
z^{\prime \prime}(x)=E(x) z^{\prime}(x)
$$

Explicitly, they are given by

$$
\begin{aligned}
\overline{\tilde{H}}^{ \pm}= & -A(z) \frac{\mathrm{d}^{2}}{\mathrm{~d} z^{2}}+\left[\frac{\mathcal{N}-2}{2} A^{\prime}(z) \pm Q(z)\right] \frac{\mathrm{d}}{\mathrm{d} z} \\
& -\left[\frac{(\mathcal{N}-1)(\mathcal{N}-2)}{12} A^{\prime \prime}(z) \pm \frac{\mathcal{N}-1}{2} Q^{\prime}(z)+R\right],
\end{aligned}
$$

where the new functions $A(z)$ and $Q(z)$ are defined by

$$
2 A(z)=z^{\prime}(x)^{2}, \quad Q(z)=-z^{\prime}(x) W(x) .
$$

The conditions (12) and (13) for type A $\mathcal{N}$-fold SUSY are reduced to the following simple forms in terms of $z$ :

$$
\begin{aligned}
& \frac{\mathrm{d}^{3}}{\mathrm{~d} z^{3}} Q(z)=0 \quad \text { for } \quad \mathcal{N} \geq 2 \\
& \frac{\mathrm{d}^{5}}{\mathrm{~d} z^{5}} A(z)=0 \quad \text { for } \quad \mathcal{N} \geq 3 .
\end{aligned}
$$

In particular, the condition $(21)$ indicates that $Q(z)$ is a polynomial of at most second-degree in $z$ for all $\mathcal{N} \geq 2$ :

$$
Q(z)=b_{2} z^{2}+b_{1} z+b_{0}
$$

In terms of $z(x)$, the potential terms $V^{ \pm}(x)$ of type A $\mathcal{N}$-fold SUSY Hamiltonians in (10) are expressed as

$$
\begin{aligned}
V^{ \pm}(x)=-\frac{1}{12 A(z)}\left[\left(\mathcal{N}^{2}-1\right)\left(A(z) A^{\prime \prime}(z)-\frac{3}{4} A^{\prime}(z)^{2}\right)-3 Q(z)^{2}\right. & \\
& \left.\mp 3 \mathcal{N}\left(A^{\prime}(z) Q(z)-2 A(z) Q^{\prime}(z)\right)\right]-\left.R\right|_{z=z(x)}
\end{aligned}
$$


The type A $\mathcal{N}$-fold SUSY systems (10) and (11) have an underlying symmetry which, as we shall show, plays a central role in investigating the existence of intermediate Hamiltonians. It is $G L(2, \mathbb{C})$ linear fractional transformations on the variable $z$ introduced as

$$
z=\frac{\alpha w+\beta}{\gamma w+\delta} \quad(\alpha, \beta, \gamma, \delta \in \mathbb{C}, \Delta \equiv \alpha \delta-\beta \gamma \neq 0) .
$$

Then, the type A monomial space is invariant under the $G L(2, \mathbb{C})$ transformations induced by $(25)$ :

$$
\tilde{\mathcal{V}}_{\mathcal{N}}^{(\mathrm{A})}[z] \mapsto \widehat{\tilde{\mathcal{V}}}_{\mathcal{N}}^{(\mathrm{A})}[w]=\left.(\gamma w+\delta)^{\mathcal{N}-1} \tilde{\mathcal{V}}_{\mathcal{N}}^{(\mathrm{A})}[z]\right|_{z=\frac{\alpha w+\beta}{\gamma w+\delta}}=\tilde{\mathcal{V}}_{\mathcal{N}}^{(\mathrm{A})}[w]
$$

The gauged Hamiltonians $\tilde{H}^{-}$and $\bar{H}^{+}$are both such linear differential operators that preserve the type A monomial space, as was shown in (17). As a consequence, they are covariant under the following $G L(2, \mathbb{C})$ transformations:

$$
\overline{\tilde{H}}^{ \pm}[z] \mapsto \widehat{\tilde{\tilde{H}}}^{ \pm}[w]=\left.(\gamma w+\delta)^{\mathcal{N}-1} \overline{\tilde{H}}^{ \pm}[z](\gamma w+\delta)^{-(\mathcal{N}-1)}\right|_{z=\frac{\alpha w+\beta}{\gamma w+\delta}}
$$

that is, the transformed operators $\widehat{\widetilde{H}}^{-}$and $\widehat{\bar{H}}^{+}$both have the same forms as given in (19) with $z$ replaced by $w$ and with $A(z)$ and $Q(z)$ replaced by the transformed functions $\widehat{A}(w)$ and $\widehat{Q}(w)$ given by

$$
\begin{aligned}
& A(z) \mapsto \widehat{A}(w)=\left.\Delta^{-2}(\gamma w+\delta)^{4} A(z)\right|_{z=\frac{\alpha w+\beta}{\gamma w+\delta}}, \\
& Q(z) \mapsto \widehat{Q}(w)=\left.\Delta^{-1}(\gamma w+\delta)^{2} Q(z)\right|_{z=\frac{\alpha w+\beta}{\gamma w+\delta}}
\end{aligned}
$$

In particular, the explicit form of $\widehat{Q}(w)$ for arbitrary $\mathcal{N} \geq 2$ is given by

$$
\widehat{Q}(w)=\hat{b}_{2} w^{2}+\hat{b}_{1} w+\hat{b}_{0},
$$

with

$$
\left(\begin{array}{l}
\hat{b}_{2} \\
\hat{b}_{1} \\
\hat{b}_{0}
\end{array}\right)=\Delta^{-1}\left(\begin{array}{rrr}
\alpha^{2} & \alpha \gamma & \gamma^{2} \\
2 \alpha \beta & \alpha \delta+\beta \gamma & 2 \gamma \delta \\
\beta^{2} & \beta \delta & \delta^{2}
\end{array}\right)\left(\begin{array}{l}
b_{2} \\
b_{1} \\
b_{0}
\end{array}\right)
$$

Utilizing the transformation (29) and the formulas

$$
w(x)=-\frac{\delta z(x)-\beta}{\gamma z(x)-\alpha}, \quad w^{\prime}(x)=\frac{\Delta z^{\prime}(x)}{(\gamma z(x)-\alpha)^{2}}=\Delta^{-1}(\gamma w(x)+\delta)^{2} z^{\prime}(x),
$$

we obtain the transformations of $W(x)$ and $E(x)$ as

$$
\begin{aligned}
W(x) & \mapsto \widehat{W}(x)=-\frac{\widehat{Q}(w)}{w^{\prime}(x)}=-\frac{Q(z)}{z^{\prime}(x)}=W(x), \\
E(x) & \mapsto \widehat{E}(x)=\frac{w^{\prime \prime}(x)}{w^{\prime}(x)}=\frac{z^{\prime \prime}(x)}{z^{\prime}(x)}-\frac{2 \gamma z^{\prime}(x)}{\gamma z(x)-\alpha}=E(x)-\frac{2 \gamma z^{\prime}(x)}{\gamma z(x)-\alpha} .
\end{aligned}
$$


The invariance of the pair of type A $\mathcal{N}$-fold SUSY Hamiltonians $H^{ \pm}$under the $G L(2, \mathbb{C})$ transformations also follows from a direct application of (33) and (34):

$$
H^{ \pm}[W, E]=H^{ \pm}[\widehat{W}, \widehat{E}]
$$

since $\widehat{W}(x)=W(x)$ and from (18) and (34) we have

$$
2 \widehat{E}^{\prime}(x)-\widehat{E}(x)^{2}=2 E^{\prime}(x)-E(x)^{2} .
$$

On the other hand, the invariance of the type A $\mathcal{N}$-fold supercharge $P_{\mathcal{N}}^{-}$is not manifest in the factorized form (11) and due to the fact that $\widehat{E}(x) \neq E(x)$ the factorized form is in appearance not invariant:

$$
\begin{aligned}
P_{\mathcal{N}}^{-}[\widehat{W}, \widehat{E}] & =\prod_{k=0}^{\mathcal{N}-1}\left(\frac{\mathrm{d}}{\mathrm{d} x}+\widehat{W}(x)+\frac{\mathcal{N}-1-2 k}{2} \widehat{E}(x)\right) \\
& =\prod_{k=0}^{\mathcal{N}-1}\left(\frac{\mathrm{d}}{\mathrm{d} x}+W(x)+\frac{\mathcal{N}-1-2 k}{2}\left(E(x)-\frac{2 \gamma z^{\prime}(x)}{\gamma z(x)-\alpha}\right)\right) .
\end{aligned}
$$

The fact that $P_{\mathcal{N}}^{-}$is also invariant under the $G L(2, \mathbb{C})$ transformations

$$
P_{\mathcal{N}}^{-}[\widehat{W}, \widehat{E}]=P_{\mathcal{N}}^{-}[W, E]
$$

proved in Ref. [25], despite the non-invariance in appearance for $\gamma \neq 0$, indicates that the type A $\mathcal{N}$-fold supercharge admits a one-parameter family of different factorizations characterized by the parameter $\alpha / \gamma$.

\section{INTERMEDIATE HAMILTONIANS FOR $\mathcal{N}=2$}

From now on, we shall restrict ourselves to the case of $\mathcal{N}=2$. The type A $\mathcal{N}$-fold SUSY systems (10) and (11) for $\mathcal{N}=2 \mathrm{read}$

$$
\begin{gathered}
2 H^{ \pm}=-\frac{\mathrm{d}^{2}}{\mathrm{~d} x^{2}}+W(x)^{2}-\frac{E^{\prime}(x)}{2}+\frac{E(x)^{2}}{4}-2 R \pm 2 W^{\prime}(x), \\
P_{2}^{-}=P_{21}^{-} P_{22}^{-}=\frac{\mathrm{d}^{2}}{\mathrm{~d} x^{2}}+2 W(x) \frac{\mathrm{d}}{\mathrm{d} x}+W^{\prime}(x)+W(x)^{2}+\frac{E^{\prime}(x)}{2}-\frac{E(x)^{2}}{4},
\end{gathered}
$$

where

$$
P_{21}^{-}=\frac{\mathrm{d}}{\mathrm{d} x}+W(x)-\frac{E(x)}{2}, \quad P_{22}^{-}=\frac{\mathrm{d}}{\mathrm{d} x}+W(x)+\frac{E(x)}{2} .
$$

The superHamiltonian $\boldsymbol{H}_{2}$ and the type A 2-fold supercharges $\boldsymbol{Q}_{2}^{ \pm}$introduced with the ordinary fermionic variables $\psi^{ \pm}$as

$$
\boldsymbol{H}_{2}=H^{-} \psi^{-} \psi^{+}+H^{+} \psi^{+} \psi^{-}, \quad \boldsymbol{Q}_{2}^{ \pm}=P_{2}^{\mp} \psi^{ \pm},
$$

satisfy the type A 2-fold superalgebra [25]:

$$
\left[\boldsymbol{Q}_{2}^{ \pm}, \boldsymbol{H}_{2}\right]=\left\{\boldsymbol{Q}_{2}^{ \pm}, \boldsymbol{Q}_{2}^{ \pm}\right\}=0, \quad\left\{\boldsymbol{Q}_{2}^{-}, \boldsymbol{Q}_{2}^{+}\right\}=4\left(\boldsymbol{H}_{2}+R\right)^{2}+4 b_{0} b_{2}-b_{1}^{2} .
$$


In the expanded form of the type A 2-fold supercharge components (40), its invariance under the $G L(2, \mathbb{C})$ transformations is now manifest by applying $(33)$ and $(36)$ :

$$
P_{2}^{-}[\widehat{W}, \widehat{E}]=P_{2}^{-}[W, E]
$$

However, each factor of the type A $\mathcal{N}$-fold supercharge in the factorized form is not invariant since $\widehat{E}(x) \neq E(x)$ as shown in (37), and thus we generally have

$$
\widehat{P}_{21}^{-} \equiv P_{21}^{-}[\widehat{W}, \widehat{E}] \neq P_{21}^{-}[W, E], \quad \widehat{P}_{22}^{-} \equiv P_{22}^{-}[\widehat{W}, \widehat{E}] \neq P_{22}^{-}[W, E] .
$$

Next, we introduce another Hamiltonian $H^{\mathrm{i} 1}$, which we shall call an intermediate Hamiltonian, as

$$
P_{22}^{-} H^{-}=H^{\mathrm{i} 1} P_{22}^{-}, \quad P_{21}^{-} H^{\mathrm{i} 1}=H^{+} P_{21}^{-},
$$

which are compatible with (15). It is evident that $H^{\mathrm{i} 1}$ is in general not invariant under the $G L(2, \mathbb{C})$ transformation in contrast with $H^{ \pm}$due to the fact that both of $P_{21}^{-}$and $P_{22}^{-}$ which intertwine $H^{\mathrm{i} 1}$ with $H^{ \pm}$have no invariance (45). Hence, we can expect a family of intermediate Hamiltonians for each given type A 2-fold SUSY system. Needless to say, however, we do not always have such an intermediate Hamiltonian for a given system. The necessary and sufficient conditions for its existence are that there exist two constants $C_{22}$ and $C_{21}$ such that $H^{ \pm}$and $H^{\mathrm{i} 1}$ are expressed as (see, e.g., Refs. [2, 3, 4])

$$
\begin{aligned}
& 2 H^{-}=P_{22}^{+} P_{22}^{-}+2 C_{22}, \quad 2 H^{+}=P_{21}^{-} P_{21}^{+}+2 C_{21}, \\
& 2 H^{\mathrm{i} 1}=P_{22}^{-} P_{22}^{+}+2 C_{22}=P_{21}^{+} P_{21}^{-}+2 C_{21},
\end{aligned}
$$

where $P_{i j}^{+}$are the transpositions of $P_{i j}^{-}$, that is, ${ }^{2}$

$$
P_{21}^{+}=-\frac{\mathrm{d}}{\mathrm{d} x}+W(x)-\frac{E(x)}{2}, \quad P_{22}^{+}=-\frac{\mathrm{d}}{\mathrm{d} x}+W(x)+\frac{E(x)}{2} .
$$

More explicitly, the conditions (47) read

$$
\begin{aligned}
2 H^{-} & =-\frac{\mathrm{d}^{2}}{\mathrm{~d} x^{2}}+W(x)^{2}+E(x) W(x)+\frac{E(x)^{2}}{4}-\frac{E^{\prime}(x)}{2}-W^{\prime}(x)+2 C_{22}, \\
2 H^{\mathrm{i} 1} & =-\frac{\mathrm{d}^{2}}{\mathrm{~d} x^{2}}+W(x)^{2}+E(x) W(x)+\frac{E(x)^{2}}{4}+\frac{E^{\prime}(x)}{2}+W^{\prime}(x)+2 C_{22} \\
& =-\frac{\mathrm{d}^{2}}{\mathrm{~d} x^{2}}+W(x)^{2}-E(x) W(x)+\frac{E(x)^{2}}{4}+\frac{E^{\prime}(x)}{2}-W^{\prime}(x)+2 C_{21}, \\
2 H^{+} & =-\frac{\mathrm{d}^{2}}{\mathrm{~d} x^{2}}+W(x)^{2}-E(x) W(x)+\frac{E(x)^{2}}{4}-\frac{E^{\prime}(x)}{2}+W^{\prime}(x)+2 C_{21} .
\end{aligned}
$$

From Eqs. (39) and (49), the necessary and sufficient conditions reduce to

$$
W^{\prime}(x)+E(x) W(x)=-2 R-2 C_{22}=C_{21}-C_{22}=2 C_{21}+2 R .
$$

\footnotetext{
${ }^{2}$ Note that we do not assume the reality of the functions $W(x)$ and $E(x)$.
} 
Noting the relation

$$
W^{\prime}(x)+E(x) W(x)=-Q^{\prime}(z),
$$

which easily follows from (18) and (20), we find that the latter conditions (50) are equivalent to

$$
Q(z)=\left(C_{22}-C_{21}\right) z+b_{0}, \quad-2 R=C_{22}+C_{21},
$$

with $b_{0}$ being another constant. We recall that for the most general type A $\mathcal{N}$-fold SUSY systems for all $\mathcal{N} \geq 2, Q(z)$ is given by a polynomial of at most second-degree (23). Hence, a given type A 2-fold SUSY system (39) admits an intermediate Hamiltonian $H^{\mathrm{il}}$ satisfying Eq. (46) if and only if

$$
b_{2}=0, \quad b_{1}=C_{22}-C_{21}, \quad-2 R=C_{22}+C_{21} .
$$

The last two conditions in (53) just determine these constants for the given values of $b_{1}$ and $R$. Hence, only the first condition in (53) is essential for the existence of an intermediate Hamiltonian.

As was discussed previously, the type A 2-fold supercharge $P_{2}^{-}$is invariant under the $G L(2, \mathbb{C})$ transformation (44) while its factors $P_{22}^{-}$and $P_{21}^{-}$are not (45) for $\gamma \neq 0$. As a consequence, the necessary and sufficient conditions (53) for the existence of another intermediate Hamiltonian $H^{\mathrm{i} 2}$ after a $G L(2, \mathbb{C})$ transformation are accordingly changed as

$$
\hat{b}_{2}=0, \quad \hat{b}_{1}=\hat{C}_{22}-\hat{C}_{21}, \quad-2 R=\hat{C}_{22}+\hat{C}_{21},
$$

where $\hat{C}_{22}$ and $\hat{C}_{21}$ are another set of constants. Again, only the first condition in (54) is essential for the existence of another intermediate Hamiltonian $H^{\mathrm{i} 2}$ after the transformation. Under the fulfillment of the conditions (54), the original type A 2-fold SUSY Hamiltonians $H^{ \pm}$and the new intermediate Hamiltonian $H^{\mathrm{i} 2}$ are expressed in terms of the transformed supercharges as

$$
\begin{aligned}
& 2 H^{-}=\widehat{P}_{22}^{+} \widehat{P}_{22}^{-}+2 \hat{C}_{22}, \quad 2 H^{+}=\widehat{P}_{21}^{-} \widehat{P}_{21}^{+}+2 \hat{C}_{21}, \\
& 2 H^{\mathrm{i} 2}=\widehat{P}_{22}^{-} \widehat{P}_{22}^{+}+2 \hat{C}_{22}=\widehat{P}_{21}^{+} \widehat{P}_{21}^{-}+2 \hat{C}_{21},
\end{aligned}
$$

where $\widehat{P}_{i j}^{+}$are the transpositions of $\widehat{P}_{i j}^{-}$which were defined in (45), that is,

$$
\widehat{P}_{21}^{ \pm}=\mp \frac{\mathrm{d}}{\mathrm{d} x}+\widehat{W}(x)-\frac{\widehat{E}(x)}{2}, \quad \widehat{P}_{22}^{ \pm}=\mp \frac{\mathrm{d}}{\mathrm{d} x}+\widehat{W}(x)+\frac{\widehat{E}(x)}{2} .
$$

It is now clear that a type A 2-fold SUSY system which satisfies the conditions (53) and thus admits an intermediate Hamiltonian $H^{\mathrm{i} 1}$ also admits another different intermediate Hamiltonian $H^{\mathrm{i} 2}$ after a $G L(2, \mathbb{C})$ transformation with $\gamma \neq 0$ if and only if the conditions (54) are simultaneously fulfilled in addition to (53). It essentially means the satisfaction of $b_{2}=\hat{b}_{2}=0$. From the transformation formula (31) we immediately see that it is only possible for the $G L(2, \mathbb{C})$ transformation with $\gamma \neq 0$ which satisfies

$$
\alpha b_{1}+\gamma b_{0}=0 .
$$




\begin{tabular}{cc}
\hline Conditions & Number of $H^{\mathrm{i}}$ \\
\hline$b_{2} \neq 0$ & 0 \\
$b_{2}=b_{1}=0$ & 1 \\
$b_{2}=0, b_{1} \neq 0$ & 2 \\
\hline
\end{tabular}

TABLE I: The admissible numbers of different intermediate Hamiltonians in type A 2-fold supersymmetry.

Let us first consider the case when $b_{1}=0$. In this case we can assume that $b_{0} \neq 0$; otherwise $Q(z)=0$ since $b_{2}=0$ is already assumed in order to meet the condition (53), and from $(24) V^{-}(x)=V^{+}(x)$ which means that the system is trivial as 2-fold SUSY. But for $b_{1}=0$ and $b_{0} \neq 0$ there is no solution to the equation (57) except for $\gamma=0$. But for any $G L(2, \mathbb{C})$ transformation with $\gamma=0$, the function $E(x)$ is invariant by Eq. (34) and so is the intermediate Hamiltonian $H^{\mathrm{i} 1}$. Hence in the case of $b_{1}=0$ the factorization of type A 2-fold supercharge admitting intermediate Hamiltonians is unique. On the other hand, in the case of $b_{1} \neq 0$ the solution to the equation (57) is given by

$$
\alpha / \gamma=-b_{0} / b_{1}
$$

As was shown previously, any type A 2-fold supercharge admits one-parameter family of factorizations $P_{2}^{-}=P_{21}^{-} P_{22}^{-}$characterized by the parameter $\alpha / \gamma$. In addition, any type $\mathrm{A}$ 2-fold SUSY system with $b_{2}=0$ has at least one intermediate Hamiltonian $H^{\mathrm{i} 1}$. Then, the result (58) tells us that if the system further satisfies $b_{1} \neq 0$, it can admit a one and only one additional and different intermediate Hamiltonian $H^{\mathrm{i} 2}$ at the one point (58) in the parameter space of $\alpha / \gamma \in \mathbb{C}$. In Table I, we summarize the results.

\section{SECOND-ORDER PARASUPERSYMMETRY}

In the previous section, we have just verified that a type A 2-fold SUSY system $\left(H^{ \pm}, P_{2}^{-}=\right.$ $P_{21}^{-} P_{22}^{-}$) admits (at least) one intermediate Hamiltonian $H^{\mathrm{i} 1}$ if and only if the condition $b_{2}=0$ holds. In this section, we shall further show that any such a system can possess an additional symmetry, namely, parasupersymmetry of order 2 introduced in Ref. [28]. Indeed, for a given such type A 2-fold SUSY system we can define a triple of operators $\left(\boldsymbol{H}_{\mathrm{P}}, \boldsymbol{Q}_{\mathrm{P}}^{ \pm}\right)$by

$$
\begin{aligned}
& \boldsymbol{H}_{\mathrm{P}}=H^{-}\left(\psi_{\mathrm{P}}^{-}\right)^{2}\left(\psi_{\mathrm{P}}^{+}\right)^{2}+H^{\mathrm{i} 1}\left(\psi_{\mathrm{P}}^{+} \psi_{\mathrm{P}}^{-}-\left(\psi_{\mathrm{P}}^{+}\right)^{2}\left(\psi_{\mathrm{P}}^{-}\right)^{2}\right)+H^{+}\left(\psi_{\mathrm{P}}^{+}\right)^{2}\left(\psi_{\mathrm{P}}^{-}\right)^{2}, \\
& \boldsymbol{Q}_{\mathrm{P}}^{-}=P_{22}^{+}\left(\psi_{\mathrm{P}}^{-}\right)^{2} \psi_{\mathrm{P}}^{+}+P_{21}^{+} \psi_{\mathrm{P}}^{+}\left(\psi_{\mathrm{P}}^{-}\right)^{2}, \quad \boldsymbol{Q}_{\mathrm{P}}^{+}=P_{22}^{-} \psi_{\mathrm{P}}^{-}\left(\psi_{\mathrm{P}}^{+}\right)^{2}+P_{21}^{-}\left(\psi_{\mathrm{P}}^{+}\right)^{2} \psi_{\mathrm{P}}^{-},
\end{aligned}
$$

where $\psi_{\mathrm{P}}^{ \pm}$are parafermions of order 2 satisfying [29]

$$
\left(\psi_{\mathrm{P}}^{ \pm}\right)^{2} \neq 0, \quad\left(\psi_{\mathrm{P}}^{ \pm}\right)^{3}=0, \quad\left\{\psi_{\mathrm{P}}^{-}, \psi_{\mathrm{P}}^{+}\right\}+\left\{\left(\psi_{\mathrm{P}}^{-}\right)^{2},\left(\psi_{\mathrm{P}}^{+}\right)^{2}\right\}=2 I
$$

Then, using Eq. (47) and the parafermionic algebra of order 2 in Ref. [29] we can show that the triple $\left(\boldsymbol{H}_{\mathrm{P}}, \boldsymbol{Q}_{\mathrm{P}}^{ \pm}\right)$defined as (59) satisfies the second-order paraSUSY relations in Ref. [28]:

$$
\begin{array}{r}
\left(\boldsymbol{Q}_{\mathrm{P}}^{ \pm}\right)^{2} \neq 0, \quad\left(\boldsymbol{Q}_{\mathrm{P}}^{ \pm}\right)^{3}=0, \quad\left[\boldsymbol{Q}_{\mathrm{P}}^{ \pm}, \boldsymbol{H}_{\mathrm{P}}\right]=0, \\
\left(\boldsymbol{Q}_{\mathrm{P}}^{ \pm}\right)^{2} \boldsymbol{Q}_{\mathrm{P}}^{\mp}+\boldsymbol{Q}_{\mathrm{P}}^{ \pm} \boldsymbol{Q}_{\mathrm{P}}^{\mp} \boldsymbol{Q}_{\mathrm{P}}^{ \pm}+\boldsymbol{Q}_{\mathrm{P}}^{\mp}\left(\boldsymbol{Q}_{\mathrm{P}}^{ \pm}\right)^{2}=4 \boldsymbol{Q}_{\mathrm{P}}^{ \pm} \boldsymbol{H}_{\mathrm{P}},
\end{array}
$$


if and only if the constants $C_{i j}$ in (47) satisfy

$$
C_{22}=-C_{21}=b_{1} / 2
$$

and thus in particular $R=0$ by (53). Hence, we conclude that any type A 2-fold SUSY quantum system with (at least) one intermediate Hamiltonian also has paraSUSY of order 2 when $R=0$. The additional restriction $R=0$ arises since one of the paraSUSY conditions (62) is not invariant under any constant shift of $\boldsymbol{H}_{\mathrm{P}}$. Furthermore, as was shown in Ref. [29] this type of realization of second-order paraSUSY systems admits an additional novel nonlinear relation as the following (cf., Eq. (6.66) in the latter reference):

$$
\left(\boldsymbol{Q}_{\mathrm{P}}^{-}\right)^{2}\left(\boldsymbol{Q}_{\mathrm{P}}^{+}\right)^{2}+\boldsymbol{Q}_{\mathrm{P}}^{ \pm}\left(\boldsymbol{Q}_{\mathrm{P}}^{\mp}\right)^{2} \boldsymbol{Q}_{\mathrm{P}}^{ \pm}+\left(\boldsymbol{Q}_{\mathrm{P}}^{+}\right)^{2}\left(\boldsymbol{Q}_{\mathrm{P}}^{-}\right)^{2}=4\left(\boldsymbol{H}_{\mathrm{P}}\right)^{2}-b_{1}^{2},
$$

which can be regarded as a generalized (type A) 2-fold superalgebra. In fact, on the one hand we immediately have from the paraSUSY relations in (61)

$$
\left\{\left(\boldsymbol{Q}_{\mathrm{P}}^{ \pm}\right)^{2},\left(\boldsymbol{Q}_{\mathrm{P}}^{ \pm}\right)^{2}\right\}=0, \quad\left[\left(\boldsymbol{Q}_{\mathrm{P}}^{ \pm}\right)^{2}, \boldsymbol{H}_{\mathrm{P}}\right]=0
$$

while on the other hand the nonlinear relation (64) reduces, in the subsector with the parafermion number zero and two, to

$$
\left\{\left(\boldsymbol{Q}_{\mathrm{P}}^{-}\right)^{2},\left(\boldsymbol{Q}_{\mathrm{P}}^{+}\right)^{2}\right\}=4\left(\boldsymbol{H}_{\mathrm{P}}\right)^{2}-b_{1}^{2} .
$$

Then, the commutation and anti-commutation relations (65) and (66) are, under the assumed condition $b_{2}=0$ and $R=0$, entirely identical with the type A 2-fold superalgebra (43) with the trivial identification of the type A 2-fold supercharges $\boldsymbol{Q}_{2}^{ \pm}$with $\left(\boldsymbol{Q}_{\mathrm{P}}^{ \pm}\right)^{2}$ and with the observation that in the subsector $\boldsymbol{H}_{\mathrm{P}}$ is essentially identical with $\boldsymbol{H}_{2}$. The relation between type A 2-fold SUSY and second-order paraSUSY was briefly referred to in Ref. [29]. Here we have firstly shown the necessary and sufficient conditions for a type A 2-fold SUSY system to admit simultaneously second-order paraSUSY, namely, Eqs. (53) and (63). Finally, it is evident that we can construct two sets of second-order paraSUSY systems whenever a type A 2-fold SUSY system has two different intermediate Hamiltonians.

\section{AN APPLICATION TO THE GENERALIZED PÖSCHL-TELLER POTEN- TIAL}

As an application of the general framework discussed in the previous two sections, we shall reconstruct the BQR SSUSY model (1)-(9) and its generalization which preserves all the SUSY and SSUSY structure therein. To this end, let us first choose the change of variable $z=z(x)$ which determines the relation between physical Hamiltonians and gauged ones as

$$
\begin{aligned}
z(x) & =-(\sinh x)^{-2 B}\left(\tanh \frac{x}{2}\right)^{2 A+1} \\
& =-2^{-2 B}\left(\sinh \frac{x}{2}\right)^{2 A-2 B+1}\left(\cosh \frac{x}{2}\right)^{-(2 A+2 B+1)}
\end{aligned}
$$

where $A$ and $B$ are both constants. The function $A(z)=z^{\prime}(x)^{2} / 2$ defined in $(20)$ and its derivatives with respect to $z$ are in general transcendental functions of $z$. Explicitly, they 
read

$$
\begin{aligned}
z^{\prime}(x) & =-z(x) \frac{2 B \cosh x-2 A-1}{\sinh x}, \\
A(z) & =\frac{z(x)^{2}(2 B \cosh x-2 A-1)^{2}}{2 \sinh ^{2} x} \\
A^{\prime}(z) & =z(x)\left(4 B^{2}-\frac{\alpha_{1}^{+} \cosh x-\alpha_{2}^{+}}{\sinh ^{2} x}\right), \\
A^{\prime \prime}(z) & =4 B^{2}-\frac{\beta_{1} \cosh x-\beta_{2}}{\sinh ^{2} x}-\frac{2 A+1}{2 B \cosh x-2 A-1},
\end{aligned}
$$

where $\alpha_{i}^{+}$and $\beta_{i}$ are all constants given by

$$
\begin{aligned}
\alpha_{1}^{ \pm} & =(2 A+1)(4 B \pm 1), & \alpha_{2}^{ \pm} & =(2 A+1)^{2}+2 B(2 B \pm 1), \\
\beta_{1} & =(2 A+1)(4 B+3), & \beta_{2} & =(2 A+1)^{2}+2(B+1)(2 B+1) .
\end{aligned}
$$

Substituting them into the most general form of a pair of type A 2-fold SUSY potentials, Eq. (24) with $\mathcal{N}=2$, we obtain

$$
\begin{aligned}
V^{ \pm}(x)= & \frac{Q(z(x))^{2} \sinh ^{2} x}{2 z(x)^{2}(2 B \cosh x-2 A-1)^{2}}+\frac{4(2 A+1) B \cosh x+(2 A+1)^{2}-12 B^{2}}{8(2 B \cosh x-2 A-1)^{2}} \\
& -\frac{(2 A+1) B \cosh x-A(A+1)-B^{2}}{2 \sinh ^{2} x}+\frac{B^{2}}{2}-R \\
& \pm \frac{Q(z(x))\left(4 B^{2} \sinh ^{2} x-\alpha_{1}^{+} \cosh x+\alpha_{2}^{+}\right)}{z(x)(2 B \cosh x-2 A-1)^{2}} \mp Q^{\prime}(z(x)),
\end{aligned}
$$

where $Q(z)$ is a polynomial of at most second-degree given as in (23). The function $W(x)$ characterizing the type A system in this case reads

$$
W(x)=-\frac{Q(z(x))}{z^{\prime}(x)}=\frac{Q(z(x)) \sinh x}{z(x)(2 B \cosh x-2 A-1)} .
$$

The other function $E(x)$ defined through the relation (18), which also characterizes the type A system, is calculated as

$$
E(x)=\frac{z^{\prime \prime}(x)}{z^{\prime}(x)}=-\frac{(2 B+1) \cosh x-2 A-1}{\sinh x}+\frac{2 B \sinh x}{2 B \cosh x-2 A-1} .
$$

Next, let us consider the case when the type A 2-fold SUSY system admits an intermediate Hamiltonian, namely, $b_{2}=0$. From (75) and (76) we have

$$
\begin{aligned}
W^{\prime}(x)= & \frac{Q(z(x))}{z(x)}-Q^{\prime}(z(x))-\frac{(2 A+1) \cosh x-2 B}{z(x)(2 B \cosh x-2 A-1)^{2}} Q(z(x)), \\
E^{\prime}(x)= & -\frac{2(2 A+1) B \cosh x-4 B^{2}}{(2 B \cosh x-2 A-1)^{2}}-\frac{(2 A+1) \cosh x-2 B-1}{\sinh ^{2} x} \\
E(x)^{2}= & 4 B^{2}+\frac{(2 A+1)^{2}-4 B^{2}}{(2 B \cosh x-2 A-1)^{2}} \\
& -\frac{2(2 A+1)(2 B+1) \cosh x-(2 A+1)^{2}-(2 B+1)^{2}}{\sinh ^{2} x} .
\end{aligned}
$$


Substituting (75)-(79) into (49b), using the relations (53) among the constants, and noting that $Q^{\prime}(z)=b_{1}$ when $b_{2}=0$, we obtain the intermediate potential $V^{\mathrm{i} 1}(x)$ as

$$
\begin{aligned}
V^{\mathrm{i} 1}(x)= & \frac{Q(z(x))^{2} \sinh ^{2} x}{2 z(x)^{2}(2 B \cosh x-2 A-1)^{2}}-\frac{4(2 A+1) B \cosh x-(2 A+1)^{2}-4 B^{2}}{8(2 B \cosh x-2 A-1)^{2}} \\
& -\frac{(2 A+1)(B+1) \cosh x-A(A+1)-(B+1)^{2}}{2 \sinh ^{2} x}+\frac{B^{2}}{2}-R .
\end{aligned}
$$

Next, we shall consider the case when the system admits another different intermediate Hamiltonian $H^{\mathrm{i} 2}$, namely, $b_{1} \neq 0$. The $G L(2, \mathbb{C})$ transformation which takes the type A 2-fold supercharge to another factorization for which $H^{\mathrm{i} 2}$ exists must satisfy the condition (58). The parameter $\delta$ does not play an important role in our context, so we set $\delta=0$ without any loss of generality. But in this case $\beta$ cannot be 0 otherwise $\Delta=0$. Thus, we fix the parameters as

$$
\alpha / \gamma=-b_{0} / b_{1} \equiv-z_{0}, \quad \beta / \gamma=-1, \quad \delta=0 .
$$

In other words, we choose the following $G L(2, \mathbb{C})$ transformation on the variable $z(x)$ :

$$
w(x)=-\frac{1}{z(x)+z_{0}}=\frac{1}{(\sinh x)^{-2 B}(\tanh x / 2)^{2 A+1}-z_{0}} .
$$

The function $W(x)$ is invariant under the transformation (see, Eq. (33)) while $E(x)$ is transformed according to (34) as

$$
\begin{aligned}
\widehat{E}(x)= & E(x)-\frac{2 z^{\prime}(x)}{z(x)+z_{0}}=E(x)+\frac{2 z(x)}{z(x)+z_{0}} \frac{2 B \cosh x-2 A-1}{\sinh x} \\
= & \frac{\left[(2 B-1) z(x)-(2 B+1) z_{0}\right] \cosh x-(2 A+1)\left(z(x)-z_{0}\right)}{\left(z(x)+z_{0}\right) \sinh x} \\
& +\frac{2 B \sinh x}{2 B \cosh x-2 A-1} .
\end{aligned}
$$

Noting the relation $Q(z)=b_{1}\left(z+z_{0}\right)$ when $b_{2}=0$, we have

$$
\widehat{E}(x) \widehat{W}(x)=E(x) W(x)+2 b_{1} .
$$

From the second expression of $\widehat{E}(x)$ in (83), we obtain the following formulas:

$$
\begin{aligned}
\widehat{E}^{\prime}(x)= & E^{\prime}(x)+\frac{2 z(x)}{z(x)+z_{0}} \frac{(2 A+1) \cosh x-2 B}{\sinh ^{2} x} \\
& -\frac{2 z_{0} z(x)}{\left(z(x)+z_{0}\right)^{2}} \frac{(2 B \cosh x-2 A-1)^{2}}{\sinh ^{2} x} \\
\widehat{E}(x)^{2}= & E(x)^{2}+\frac{8 B z(x)}{z(x)+z_{0}}-\frac{4 z(x) \cosh x(2 B \cosh x-2 A-1)}{\left(z(x)+z_{0}\right) \sinh ^{2} x} \\
& -\frac{4 z_{0} z(x)(2 B \cosh x-2 A-1)^{2}}{\left(z(x)+z_{0}\right)^{2} \sinh ^{2} x} .
\end{aligned}
$$

Substituting (83)-(86) into Eq. (55) and noting that $\hat{b}_{1}=-b_{1}$ by the transformation formula (31) in our choice of the parameters $(81)$, we obtain for $H^{ \pm}$the same potentials as the ones 
in (74), as they should be, while for the other intermediate Hamiltonian $H^{\mathrm{i} 2}$ the following form of the potential:

$$
\begin{aligned}
V^{\mathrm{i} 2}(x)= & V^{\mathrm{i} 1}(x)+\frac{z(x)^{2}[(2 A+1) \cosh x-2 B]}{\left(z(x)+z_{0}\right)^{2} \sinh ^{2} x} \\
& +\frac{z_{0} z(x)\left(\alpha_{1}^{+} \cosh x-\alpha_{2}^{+}\right)}{\left(z(x)+z_{0}\right)^{2} \sinh ^{2} x}-\frac{4 B^{2} z_{0} z(x)}{\left(z+z_{0}\right)^{2}} .
\end{aligned}
$$

If we substitute (80) for $V^{\mathrm{i} 1}(x)$ into the above, we finally obtain the full expression of $V^{\mathrm{i} 2}(x)$ as

$$
\begin{aligned}
V^{\mathrm{i} 2}(x)= & \frac{Q(z(x))^{2} \sinh ^{2} x}{2 z(x)^{2}(2 B \cosh x-2 A-1)^{2}}-\frac{4(2 A+1) B \cosh x-(2 A+1)^{2}-4 B^{2}}{8(2 B \cosh x-2 A-1)^{2}} \\
& -\frac{(2 A+1)(B-1) \cosh x-A(A+1)-(B-1)^{2}}{2 \sinh ^{2} x}+\frac{B^{2}}{2}-R \\
& +\frac{z_{0} z(x)\left(\alpha_{1}^{-} \cosh x-\alpha_{2}^{-}\right)}{\left(z(x)+z_{0}\right)^{2} \sinh ^{2} x}-\frac{z_{0}^{2}[(2 A+1) \cosh x-2 B]}{\left(z(x)+z_{0}\right)^{2}}-\frac{4 B^{2} z_{0} z(x)}{\left(z(x)+z_{0}\right)^{2}}
\end{aligned}
$$

where $\alpha_{i}^{-}$are defined in (72). We are now in a position to show that the type A 2-fold system with the two intermediate Hamiltonians (74), (80), and (88) contains as a special case the BQR SSUSY model (1)-(9). For the purpose, let put $b_{0}=0$ and $b_{1}=b B$. In this case, $Q(z)=b B z$ and $z_{0}=b_{0} / b_{1}=0$. Then, the 2-fold SUSY pair of the potentials $(74)$ and the two intermediate potentials (80) and (88) reduce to, respectively,

$$
\begin{aligned}
V^{ \pm}(x)= & \frac{4\left(b^{2}+1\right)(2 A+1) B \cosh x-\left(b^{2}-1\right)(2 A+1)^{2}-4\left(b^{2}+3\right) B^{2}}{8(2 B \cosh x-2 A-1)^{2}} \\
& -\frac{(2 A+1) B \cosh x-A(A+1)-B^{2}}{2 \sinh ^{2} x}+\frac{b^{2}}{8}+\frac{B^{2}}{2}-R \\
& \mp b B \frac{(2 A+1) \cosh x-2 B}{(2 B \cosh x-2 A-1)^{2}},
\end{aligned}
$$

and

$$
\begin{aligned}
V^{\mathrm{i} 1}(x)= & \left(b^{2}-1\right) \frac{4(2 A+1) B \cosh x-(2 A+1)^{2}-4 B^{2}}{8(2 B \cosh x-2 A-1)^{2}} \\
& -\frac{(2 A+1)(B+1) \cosh x-A(A+1)-(B+1)^{2}}{\sinh ^{2} x}+\frac{b^{2}}{8}+\frac{B^{2}}{2}-R,
\end{aligned}
$$

and

$$
\begin{aligned}
V^{\mathrm{i} 2}(x)= & \left(b^{2}-1\right) \frac{4(2 A+1) B \cosh x-(2 A+1)^{2}-4 B^{2}}{8(2 B \cosh x-2 A-1)^{2}} \\
& -\frac{(2 A+1)(B-1) \cosh x-A(A+1)-(B-1)^{2}}{\sinh ^{2} x}+\frac{b^{2}}{8}+\frac{B^{2}}{2}-R .
\end{aligned}
$$


The components of supercharges $P_{i j}^{ \pm}$and $\widehat{P}_{i j}^{ \pm}$given by (41), (48), and (56) in this case read

$$
\begin{aligned}
& P_{21}^{ \pm}=\mp \frac{\mathrm{d}}{\mathrm{d} x}+\frac{(2 B+1) \cosh x-2 A-1}{2 \sinh x}+\frac{(b-1) B \sinh x}{2 B \cosh x-2 A-1}, \\
& P_{22}^{ \pm}=\mp \frac{\mathrm{d}}{\mathrm{d} x}-\frac{(2 B+1) \cosh x-2 A-1}{2 \sinh x}+\frac{(b+1) B \sinh x}{2 B \cosh x-2 A-1}, \\
& \widehat{P}_{21}^{ \pm}=\mp \frac{\mathrm{d}}{\mathrm{d} x}-\frac{(2 B-1) \cosh x-2 A-1}{2 \sinh x}+\frac{(b-1) B \sinh x}{2 B \cosh x-2 A-1} \\
& \widehat{P}_{22}^{ \pm}=\mp \frac{\mathrm{d}}{\mathrm{d} x}+\frac{(2 B-1) \cosh x-2 A-1}{2 \sinh x}+\frac{(b+1) B \sinh x}{2 B \cosh x-2 A-1}
\end{aligned}
$$

It is now easy to see that the BQR SSUSY model is realized when $b=-1$. Indeed, we have the following correspondences when $b=-1$ :

$$
\begin{array}{r}
2 V^{-}(x)=V_{A, B}(x)+\frac{1}{4}+B^{2}-2 R, \quad 2 V^{+}(x)=V_{A, B, \text { ext }}(x)+\frac{1}{4}+B^{2}-2 R, \\
2 V^{\mathrm{i} 1}(x)=V_{A, B+1}(x)+\frac{1}{4}+B^{2}-2 R, \quad 2 V^{\mathrm{i} 2}(x)=V_{A, B-1}(x)+\frac{1}{4}+B^{2}-2 R, \\
P_{21}^{-} \text {or } \widehat{P}_{21}^{-}=\hat{A}, \quad P_{21}^{+} \text {or } \widehat{P}_{21}^{+}=\hat{A}^{\dagger}, \quad P_{22}^{-} \text {or } \widehat{P}_{22}^{-}=\hat{B}, \quad P_{22}^{+} \text {or } \widehat{P}_{22}^{+}=\hat{B}^{\dagger},
\end{array}
$$

and in particular $P_{2}^{-}=P_{21}^{-} P_{22}^{-}=\widehat{P}_{21}^{-} \widehat{P}_{22}^{-}=\hat{A} \hat{B}$. The relations among the constants are given by

$$
\begin{array}{r}
\bar{c}=4 C_{22}=-4 C_{21}=-2 B \quad \text { or } \quad \bar{c}=4 \hat{C}_{22}=-4 \hat{C}_{21}=2 B, \\
-\tilde{E}+\frac{\bar{c}}{2}=-E-\frac{\bar{c}}{2}=B^{2}+\frac{1}{4}, \quad R=0 .
\end{array}
$$

The last equality $R=0$ means from the results in Section IV that the BQR SSUSY model also has second-order paraSUSY. We note that the reason why the BQR SSUSY model is realized as the particular case $b_{2}=b_{0}=0$ of the most general type A 2-fold SUSY is the same as the one discussed in Ref. [27], Section 5.

\section{DISCUSSION AND SUMMARY}

In this article, we have investigated in detail under what conditions type A $\mathcal{N}$-fold SUSY systems can have intermediate Hamiltonians in the case of $\mathcal{N}=2$. It turns out that although type A 2-fold supercharge admits a one-parameter family of factorization into product of two first-order linear differential operators due to the underlying $G L(2, \mathbb{C})$ symmetry, at most two different intermediate Hamiltonians are admissible. As a by product of the studies, we have also obtained the necessary and sufficient conditions for a type A 2-fold SUSY system to possess paraSUSY of order 2 as well. When it is the case, the type A 2-fold superalgebra together with the second-order parasuperalgebra constitute a generalized 2fold superalgebra. As a demonstration of the general arguments, we have constructed the generalized Pöschl-Teller potentials which are components of type A 2-fold SUSY with two intermediate Hamiltonians and reduce to the BQR SSUSY model in a particular case.

As for the concept like the reducibility in Ref. [10], the present investigations indicate that it would be more natural and useful to classify higher-order intertwining operators according 
to the existence and the number of intermediate Hamiltonians as has been done in Table I. After employing the latter classification scheme, we can further classify them according to the properties of the intermediate Hamiltonians such as Hermiticity, $\mathcal{P} \mathcal{T}$ symmetry, and so on.

Regarding the generalized Pöschl-Teller potentials constructed in Section V, it is worth noticing that the framework of $\mathcal{N}$-fold SUSY works well even when the function $A(z)$, which controls the change of variable $z=z(x)$ from the physical coordinate $x$ to the variable $z$ in the gauged space, is a transcendental function of $z$ without destroying quasi-solvability. For all $\mathcal{N} \geq 3$ cases type A $\mathcal{N}$-fold SUSY requires the additional condition (22) so that $A(z)$ is allowed to be at most a polynomial of fourth-degree in $z$, which results in the admissible change of variable to be at most an elliptic function, see, e.g., Ref. [25]. For the $\mathcal{N}=2$ case, on the other hand, there are no such restrictions and, to the best of our knowledge, our generalized Pöschl-Teller potentials are the first quasi-solvable examples where $A(z)$ is given by a transcendental function of $z$ as Eq. (69).

The analyses for $\mathcal{N}=2$ carried out in this article are easily generalized to the cases $\mathcal{N} \geq 3$, but we anticipate that richer structure could emerge for the higher $\mathcal{N}$ cases. In the case of $\mathcal{N}=3$, for instance, according to the factorization of type A 3-fold supercharge $P_{3}^{-}=$ $P_{31}^{-} P_{32}^{-} P_{33}^{-}$we can consider not only the case where intermediate Hamiltonians between $P_{31}^{-}$ and $P_{32}^{-}$and between $P_{32}^{-}$and $P_{33}^{-}$both exist, but also the cases where they exist only between the former place or only between the latter place exclusively. It is also interesting to study whether or not type A $\mathcal{N}$-fold SUSY systems for higher $\mathcal{N}$, when they have intermediate Hamiltonians, can admit another symmetry. The fact that in the case of $\mathcal{N}=2$ they have second-order paraSUSY indicates that they could have higher-order paraSUSY [30, 31] for $\mathcal{N} \geq 3$. Indeed, it was shown in Ref. [32] that a certain realization of paraSUSY of order 3 also admits a generalized 3-fold superalgebra. Hence, at least in the case of $\mathcal{N}=3$ we have a reasonable basis to expect paraSUSY as an additional symmetry. Other candidates might be quasi-paraSUSY introduced in Ref. [29] and $\mathcal{N}$-fold paraSUSY in Ref. [33].

\section{Acknowledgments}

This work (T.Tanaka) was partially supported by the National Cheng Kung University under the grant No. OUA:95-3-2-071.

[1] E. Witten, Nucl. Phys. B 188 (1981) 513.

[2] F. Cooper, A. Khare, and U. Sukhatme, Phys. Rep. 251 (1995) 267. hep-th/9405029.

[3] G. Junker, Supersymmetric Methods in Quantum and Statistical Physics (Springer, Berlin, 1996).

[4] B. K. Bagchi, Supersymmetry in Quantum and Classical Mechanics (Chapman and Hall/CRC press, Florida, 2000).

[5] E. Schrödinger, Proc. R. Irish Acad. A 46 (1940) 9.

[6] G. Darboux, Comput. Rend. Acad. Sci. 94 (1882) 1456.

[7] A. A. Andrianov, M. V. Ioffe, and V. P. Spiridonov, Phys. Lett. A 174 (1993) 273. hepth/9303005.

[8] H. Aoyama, M. Sato, and T. Tanaka, Nucl. Phys. B 619 (2001) 105. quant-ph/0106037. 
[9] A. A. Andrianov and A. V. Sokolov, Nucl. Phys. B 660 (2003) 25. hep-th/0301062.

[10] A. A. Andrianov, M. V. Ioffe, F. Cannata, and J. P. Dedonder, Int. J. Mod. Phys. A 10 (1995) 2683. hep-th/9404061.

[11] A. A. Andrianov, M. V. Ioffe, and D. N. Nishnianidze, Theor. Math. Phys. 104 (1995) 1129.

[12] C. M. Bender and S. Boettcher, Phys. Rev. Lett. 80 (1998) 5243. physics/9712001.

[13] F. Cannata, G. Junker, and J. Trost, Phys. Lett. A 246 (1998) 219. quant-ph/9805085.

[14] B. Bagchi and R. Roychoudhury, J. Phys. A: Math. Gen. 33 (2000) L1. quant-ph/9911104.

[15] M. Znojil, F. Cannata, B. Bagchi, and R. Roychoudhury, Phys. Lett. B 483 (2000) 284. hepth/0003277.

[16] B. Mielnik, J. Math. Phys. 25 (1984) 3387.

[17] D. J. Fernández C., Lett. Math. Phys. 8 (1984) 337.

[18] D. Zhu, J. Phys. A: Math. Gen. 20 (1987) 4331.

[19] C. N. Kumar, J. Phys. A: Math. Gen. 20 (1987) 5397.

[20] N. A. Alves and E. Drigo Filho, J. Phys. A: Math. Gen. 21 (1988) 3215.

[21] E. Drigo Filho, J. Phys. A: Math. Gen. 21 (1988) L1025.

[22] A. Mitra, P. K. Roy, A. Lahiri, and B. Bagchi, Int. J. Theor. Phys. 28 (1989) 911.

[23] C. Quesne, J. Phys. A: Math. Theor. 41 (2008) 392001. arXiv:0807.4087 [quant-ph].

[24] H. Aoyama, M. Sato, and T. Tanaka, Phys. Lett. B 503 (2001) 423. quant-ph/0012065.

[25] T. Tanaka, Nucl. Phys. B 662 (2003) 413. hep-th/0212276.

[26] B. Bagchi, C. Quesne, and R. Roychoudhury, Pramana J. Phys. 73 (2009) 337. arXiv:0812.1488 [quant-ph].

[27] A. González-López and T. Tanaka, J. Phys. A: Math. Gen. 39 (2006) 3715. quant-ph/0602177.

[28] V. A. Rubakov and V. P. Spiridonov, Mod. Phys. Lett. A 3 (1988) 1337.

[29] T. Tanaka, Ann. Phys. 322 (2007) 2350. hep-th/0610311.

[30] M. Tomiya, J. Phys. A: Math. Gen. 25 (1992) 4699.

[31] A. Khare, J. Phys. A: Math. Gen. 25 (1992) L749.

[32] T. Tanaka, Ann. Phys. 322 (2007) 2682. hep-th/0612263.

[33] T. Tanaka, Mod. Phys. Lett. A 22 (2007) 2191. hep-th/0611008. 